\title{
$\mathrm{JCP}$
}

\section{Utilisation of laboratory services by health workers in a district hospital in Malawi}

S O Mepham, S Bertel Squire, L Chisuwo, et al.

J Clin Pathol 2009 62: 935-938

doi: 10.1136/jcp.2009.069062

Updated information and services can be found at:

http://jcp.bmj.com/content/62/10/935.full.html

Topic collections Articles on similar topics can be found in the following collections

Tropical medicine (infectious diseases) (186 articles)

Notes

To order reprints of this article go to:

http://jcp.bmj.com/cgi/reprintform

To subscribe to Journal of Clinical Pathology go to:

http://jcp.bmj.com/subscriptions 


\title{
Utilisation of laboratory services by health workers in a district hospital in Malawi
}

\author{
S 0 Mepham, ${ }^{1}$ S Bertel Squire, ${ }^{2}$ L Chisuwo, ${ }^{3}$ J Kandulu, ${ }^{3}$ I Bates ${ }^{2}$
}

${ }^{1}$ Africa Centre for Health and Population Studies, University of KwaZulu Natal, Durban, South Africa; ${ }^{2}$ Liverpool School of Tropical Medicine, Liverpool, UK; ${ }^{3}$ Ministry of Health and Population, Lilongwe, Malawi

Correspondence to: Imelda Bates, Liverpool School of Tropical Medicine, Pembroke Place, Liverpool L3 50A, UK; ibates@liv.ac.uk

Accepted 13 June 2009

\section{ABSTRACT}

Aims: To identify priorities for improving effective use of laboratory services in a district hospital in Malawi.

Methods: A prospective observational study of clinicianpatient interactions to analyse laboratory test requesting practices and utilisation of laboratory results. The proportion of tests that was appropriately ordered, processed and ultimately influenced clinical management was used to assess effectiveness of utilisation.

Results: 420 clinical consultations between health professionals and patients were observed. 92\% of tests were ordered appropriately, $84 \%$ were processed by the laboratory and $64 \%$ of results influenced patient management. $73-79 \%$ of high-volume tests (haemoglobin, microscopy for malaria and tuberculosis) and $32 \%$ of low-volume tests influenced management.

Conclusions: $25 \%$ of commonly requested laboratory tests were not utilised effectively; because of the high volume, interventions to improve their use are likely to be cost effective. Although $68 \%$ of low-volume tests were not used efficiently, the cost of providing support for these tests in a resource-poor setting needs to be balanced against their clinical usefulness. In contrast to published information, this study shows significant underrequesting of laboratory tests that were available.

Measures to increase appropriate test requests will have implications for clinician education as well as laboratory space, budgets and staffing levels.

Laboratory services are crucial but expensive components of health systems, representing at least $4-8 \%$ of the total health budget in wealthy countries. ${ }^{1}$ Many laboratories in poorer countries have been chronically under-resourced and neglected, operating without adequate supervision or quality monitoring systems. The HIV epidemic, increases in malaria and tuberculosis burden, and the severe shortage of trained technicians, have contributed to the rising laboratory workload in many of the world's poorest countries.

Inappropriate use of the laboratory can indicate other inadequacies in health $\mathrm{care}^{23}$ and improving the clinical use of laboratory tests is a highly efficient mechanism for cost saving. ${ }^{47}$ Research into laboratory utilisation by clinicians has been conducted exclusively in high-middle income countries using predominantly inpatient data. ${ }^{2}$ Almost nothing is known about laboratory utilisation in poorer countries, where it is particularly important to optimise the use of scarce resources.

Effective utilisation of laboratory services depends on correct selection and ordering of tests and specimen collection, analysis and reporting of results by the laboratory, and interpretation and use of tests to influence patient management or public health. ${ }^{8}$ Clinicians and technicians have interdependent functions and need to work in partnership if laboratory services are to contribute to patient care.

In this prospective study we assessed whether clinicians in a district hospital in Malawi used appropriate indications for requesting laboratory services. We documented whether tests were processed by the laboratory and whether the results were used to influence patient management. We used the results to identify areas where improvements could be made. In contrast to previous studies this work was based in one of the poorest countries in the world, and used prospective data from laboratory tests requested for inpatients and outpatients. The study was part of a Ministry of Health and Population project to develop a cost-effective model laboratory service for district hospitals in Malawi.

\section{METHODS}

This study was carried out in Ntcheu district hospital in central Malawi, a hospital with 240 beds serving a population of $\sim 500000$. Most of the inpatient clinical services were provided by clinical officers, and outpatient services were provided by medical assistants. Nursing staff ensured that samples for laboratory tests were collected from patients, apart from cerebrospinal, pleural and ascitic fluids and biopsies, which were collected by clinical officers. During the study the hospital laboratory had three qualified technicians and performed $\sim 40000$ tests per year. ${ }^{9}$

\section{Test ordering by clinicians}

A clinician "observer" (SOM), who was independent of the hospital and unknown to local health workers, observed consultations between patients and health workers, without interfering with routine decision-making processes. The study was carried out during March and April 2002, towards the end of the rainy season. The observer spent 1 week in each of five wards and 1 week in the outpatient department. Every observed consultation during this time period was included in the study. For each test ordered the observer noted whether it was appropriate, whether additional tests should have been ordered, whether the sample was obtained and processed by the laboratory, and whether the result was used to influence patient management. The outcome of each test was categorised once as "appropriate indication" or "processed" or "result used appropriately" so that no test was double counted within these categories.

National guidelines for the clinical management of patients, including laboratory tests and blood transfusions (table 1) ${ }^{10}$ were used by the observer 
to determine whether indications for tests were appropriate. These guidelines had been developed by national consensus and were based on World Health Organization recommendations. Tests that were not ordered but would have been indicated according to the guidelines were also documented.

\section{Laboratory processing of test orders}

For each test the arrival of the sample in the laboratory, the availability of a test result and reasons for not processing samples were documented using workbook records.

\section{Use of test results}

Test results were recorded as "used appropriately" if they were "diagnostic or supportive of clinical diagnosis, a useful negative, or used in monitoring of treatment or disease progress". "In the case of blood transfusion the indication was appropriate if the transfusion was essential for treatment (as defined in local guidelines), requested and administered. To eliminate interobserver error all the assessments of appropriateness of indications for tests ordered (or not ordered) and for clinicians' responses to results were performed by the same observer; uncertainties were resolved through discussion with other clinicians (IB, SBS).

\section{RESULTS}

One hundred and eighty (43\%) outpatient consultations and $240(57 \%)$ inpatient consultations (total 420) were observed: $310(74 \%)$ by clinical officers, 101 (24\%) by medical assistants, 7

Table 1 Appropriate indications for laboratory investigations ${ }^{10}$

\begin{tabular}{ll}
\hline Investigation/treatment & Indication \\
\hline Malaria parasites & Mild disease: fever, rigors, chills in the absence of other
\end{tabular}
obvious causes; splenomegaly and anaemia in the under 5 s also suggestive

Severe disease: in under $5 \mathrm{~s}$ : altered consciousness, convulsions, extreme pallor and poor oral intake

In adults: as above plus acute renal failure, ARDS, DIC jaundice, hypoglycaemia; particularly at risk include pregnant women, non-immune individuals and splenectomised patients

Guidelines allow for presumptive treatment but only when microscopy is unavailable

AAFB sputum microscopy Chronic cough for more than 3 weeks

$\mathrm{Hb}$ Clinical pallor

Blood transfusion Severe anaemia $(\mathrm{Hb}<4 \mathrm{~g} / \mathrm{l})$; severe anaemia $(\mathrm{Hb}<6 \mathrm{~g} / \mathrm{l})$ PLUS haemodynamic disturbance; acute haemorrhage PLUS shock; intra-operative where necessary

Stool Diarrhoea, anaemia, possible helminthic infection

Urine Urinary tract infection, haematuria

Urinalysis Urinary tract infection, generalised oedema HIV test
reserve test kits for screening blood

Blood film Significant anaemia (but not mentioned in treatment guidelines)

White cell count and Fever of uncertain aetiology

differential

VDRL Screening test in pregnancy

Pregnancy test Suspected pregnancy

Liver function tests Suspected hepatic dysfunction

CSF

Pleural fluid

Ascitic fluid Clinically significant ascites

Acute or chronic meningism in the absence of clinically evident increased intracranial pressure

Lymph node aspiration/ Fluctuant/suppurative or solid lymphadenopathy biopsy

AAFB, acid and alcohol fast bacilli; ARDS, adult respiratory distress syndrome; CSF, cerebrospinal fluid; DIC, disseminated intravascular coagulation;

$\mathrm{Hb}$, haemoglobin; VDRL, venereal diseases reference laboratory.
$(1.5 \%)$ by nurses and $2(0.5 \%)$ by the district medical officer (table 2). The consultations generated 226 requests for laboratory tests. The most frequently requested tests (69\%) were thick blood films for malaria microscopy, sputum smears for tuberculosis, and haemoglobin estimations. Overall, $92 \%$ $(209 / 226)$ of the tests were requested appropriately, 84\% (189/ $226)$ were analysed by the laboratory and 64\% (144/226) were used appropriately (ie, they were "diagnostic or supportive of clinical diagnosis, a useful negative, or used in monitoring treatment or disease progress") (table 3).

\section{Effectiveness of laboratory utilisation for frequently and infrequently requested services}

It was found that $94-100 \%$ of the most frequently requested tests were ordered appropriately, 90-98\% were received and analysed by the laboratory and $73-79 \%$ of results were used appropriately. The reasons for inefficient use of these tests were an inappropriate clinical response to the result (10/173), failure of the specimens to be taken or sent to the laboratory (12/173), and an inappropriate test request (11/173). Forty-four per cent (8/18) of requests for blood for transfusion did not influence management because the request was not in accordance with local guidelines. Sixty-eight per cent $(36 / 53)$ of the less frequently requested tests failed to influence management because the specimens were not taken, not received or processed by the laboratory $(24 / 53)$, the patients were unavailable $(2 / 53)$ or because the request or the response to the result was inappropriate $(10 / 53)$ (table 4$)$.

\section{Tests indicated but not requested (table 5)}

The consultations should have generated 411 tests but only 226 were actually requested. For the most common tests only $68 \%$ of tests indicated were actually requested. The major discrepancies were in malaria and HIV test requests: 38 patients were treated presumptively for malaria without a blood film examination, and 30 patients with suspected HIV did not have a confirmatory test because the limited supply of HIV test kits was used for screening potential blood donors. Only 34\% of the less common tests that were indicated were actually ordered (53/155) predominantly due to under-requesting of peripheral blood morphology, white cell counts, blood glucose, and analysis of stool, urine and cerebrospinal fluid (CSF).

\section{DISCUSSION}

To our knowledge this is the first study to prospectively analyse the utilisation of laboratory services by clinicians in a developing country. We have shown that in this setting over half of all clinical consultations generated orders for laboratory tests and that almost all of the indications for these tests (92\%) were appropriate according to local guidelines. The laboratory received samples for, and processed, at least $90 \%$ of tests for malaria, tuberculosis and haemoglobin estimation. The quality

Table 2 Location of observed clinician-patient consultations

\begin{tabular}{lc}
\hline Location & No. of observations \\
\hline Male ward & 55 \\
Female ward & 61 \\
Tuberculosis ward & 40 \\
Maternity & 23 \\
Paediatrics & 61 \\
Adult outpatients & 103 \\
Paediatric outpatients & 77 \\
Total & 420 \\
\hline
\end{tabular}


Table 3 Outcome of test requests

\begin{tabular}{lllccc}
\hline Test & $\begin{array}{l}\text { Person responsible for } \\
\text { collecting sample }\end{array}$ & $\begin{array}{l}\text { No. of tests requested } \\
\text { by clinician }\end{array}$ & $\begin{array}{l}\text { Tests requested } \\
\text { appropriately (\%) }\end{array}$ & $\begin{array}{l}\text { Tests analysed (\%) } \\
\text { That influenced management (\%) }\end{array}$ \\
\hline Malaria microscopy & Nurse & 79 & $74(94)$ & $71(90)$ & $58(73)$ \\
Sputum smear microscopy for & Nurse & 42 & $42(100)$ & $41(98)$ & $32(76)$ \\
AAFB & Nurse & 34 & $33(97)$ & $32(94)$ & $27(79)$ \\
Haemoglobin & Nurse & 18 & $13(72)$ & $17(94)$ & $10(56)$ \\
Unit of blood for transfusion & Nurse & 28 & $26(93)$ & $17(61)$ & $9(32)$ \\
Others* & Clinical officer & 25 & $21(84)$ & $11(44)$ & $8(32)$ \\
Others $\dagger$ & & 226 & $209(92)$ & $189(84)$ & $144(64)$ \\
\hline Total, (mean percentage) & & & \\
\hline
\end{tabular}

*Urine and stool microscopy, urinalysis, white blood count, glucose, venereal diseases reference laboratory, pregnancy tests, liver function tests.

$\dagger$ Cerebrospinal, ascitic and pleural fluid analysis, lymph node biopsy.

AAFB, acid and alcohol fast bacilli.

of service provided by the laboratory is likely to have been positively influenced by recent training of staff and introduction of quality assurance processes. ${ }^{9}$ Sputum smear microscopy for tuberculosis was the most effectively utilised test, reflecting the input of Malawi's national tuberculosis programme to training and supervisory visits to district hospitals.

\section{Reasons for ineffective utilisation of laboratory services}

The main reasons for ineffective utilisation of high-volume tests (malaria, tuberculosis and haemoglobin tests) were due to specimens not being taken as well as misinterpretation or inappropriate utilisation of laboratory results by clinicians. One of the most ineffective services was blood transfusion; this was of particular concern as this is a major cost component of laboratory services $^{9}$ and is associated with significant risks of transfusion reactions and infections. The reasons for the problems in transfusion practice are likely to be related to the subjective nature of the decision to transfuse as the transfusion guidelines combine qualitative clinical indications with haemoglobin estimations, and also to difficulties in obtaining blood for emergency transfusion. Some of these supply problems are being alleviated by Malawi's National Blood Transfusion Service, which supplements the family replacement donor system in Ntcheu.

Low-volume tests were utilised least effectively. Less than half of stool and urine specimens requested were collected probably because the samples are unpleasant to handle, there was a shortage of specimen containers, and there was lack of communication between the clinical staff (who ordered the tests) and nursing staff (who collected the samples). For other tests, such as HIV diagnostic testing, shortage of reagents prevented the laboratory from processing the samples. Samples that required an invasive procedure to be undertaken, such as CSF, were often not obtained.

Strategies to improve effective utilisation of laboratory services Overall only $64 \%$ of laboratory tests were effective (ie, requested appropriately, analysed and used to influence management). Ineffectiveness was due to difficulties within the laboratory $(8 \%)$, pre-analytical problems (8\%) and inappropriate use of results $(20 \%)$. A quarter of results for common tests were not used appropriately. It is the laboratory's responsibility to present results in a format that is understandable so that clinicians can convert this into useful and applicable knowledge. ${ }^{12}$ Improving the way that laboratory results are reported by the technicians and educating clinicians about the clinical use of results would therefore help to improve effective utilisation of laboratory services. Clinical supervision and reorganisation of workflow so that invasive procedures are performed in the emergency room prior to transfer to the ward would improve the collection of clinician-dependent samples such as CSF.

\section{Implications of under-requesting of laboratory tests}

One of the most surprising findings of our study was the high number of tests that were not requested despite being available in the laboratory and recommended in local guidelines. This applied particularly to malaria films, peripheral blood films for cell morphology, HIV serology, and urine and stool microscopy. It is important to restrict malaria treatment to only those with proven infection, but presumptive treatment of malaria was common although microscopy was available..$^{14}$ Since this study was carried out, HIV testing has become increasingly available for diagnosis. Lack of knowledge about the use of

Table 4 Reasons for ineffective use of test results

\begin{tabular}{|c|c|c|c|c|c|c|c|}
\hline \multirow[b]{2}{*}{ Test } & \multirow[b]{2}{*}{$\begin{array}{l}\text { Person } \\
\text { responsible for } \\
\text { obtaining } \\
\text { specimen }\end{array}$} & \multirow[b]{2}{*}{$\begin{array}{l}\text { No. of tests which } \\
\text { failed to influence } \\
\text { management (\%) }\end{array}$} & \multicolumn{5}{|l|}{ Reason } \\
\hline & & & $\begin{array}{l}\text { Inappropriate test } \\
\text { request }\end{array}$ & $\begin{array}{l}\text { Sample not taken, } \\
\text { sent or processed }\end{array}$ & $\begin{array}{l}\text { Result arrived } \\
\text { too late to } \\
\text { influence clinical } \\
\text { management }\end{array}$ & $\begin{array}{l}\text { Patient died or } \\
\text { self-discharged }\end{array}$ & $\begin{array}{l}\text { Inappropriate clinical } \\
\text { response to result }\end{array}$ \\
\hline Malaria microscopy & Nurse & $21 / 79$ (27) & 3 & $8(7 \dagger+19)$ & 2 & 2 & $5(4-9+1 \dagger \dagger)$ \\
\hline $\begin{array}{l}\text { Sputum smear for } \\
\text { tuberculosis }\end{array}$ & Nurse & $10 / 42(24)$ & 0 & $1 \$$ & 0 & 4 & $5+t$ \\
\hline Others ${ }^{*}$ & Nurse & $19 / 28(68)$ & 4 & $11 \%$ & 0 & 1 & $3+t$ \\
\hline Others $\dagger$ & Clinician & $17 / 25(68)$ & 0 & $13\left(9 \ddagger+3 \S+1^{* *}\right)$ & 0 & 1 & $3(1 \dagger \dagger+1 \dagger++1 \S \S)$ \\
\hline Total & & $82 / 226(36)$ & 19 & 36 & 2 & 14 & 22 \\
\hline
\end{tabular}

*Urine and stool microscopy, urinalysis, white blood count, glucose, venereal diseases reference laboratory, pregnancy tests, liver function tests; $\dagger$ cerebrospinal, ascitic and pleural fluid analysis, lymph node biopsy; $\$$ sample not taken; §sample not sent; 9 test not written on form so sample not processed; ** sample too old to be processed; ††test not diagnostic and no further action taken to determine diagnosis; tffailed procedure; $\S \S$ incorrect interpretation of result; $\uparrow$ clinician disbelieved result. 
Table 5 Tests indicated but not requested

\begin{tabular}{lcc}
\hline Test & No. of tests requested & Total tests indicated \\
\hline Malaria & 79 & 117 \\
AAFB & 42 & 47 \\
Hb & 34 & 43 \\
Cross match & 18 & 19 \\
HIV & 0 & 30 \\
Blood film & 0 & 26 \\
Urine microscopy & 7 & 11 \\
Urinalysis & 5 & 23 \\
Stool microscopy & 5 & 22 \\
Glucose & 2 & 20 \\
White blood count & 3 & 11 \\
VDRL & 5 & 5 \\
Pregnancy test & 0 & 2 \\
Liver function tests & 1 & 1 \\
CSF & 10 & 18 \\
Ascitic tap & 6 & 7 \\
Pleural tap & 7 & 7 \\
Lymph node biopsy/ & 2 & 2 \\
aspiration & & 411 \\
Total & 226 &
\end{tabular}

Percentage of tests indicated but not requested $=45 \%$.

AAFB, acid and alcohol fast bacilli; CSF, cerebrospinal fluid; Hb, haemoglobin; VDRL, venereal diseases reference laboratory.

laboratory tests, insufficient resources and low clinician confidence in the reliability and quality of laboratory services may all contribute to suboptimal test requesting.

\section{Strengths and limitations}

Previous studies analysing clinical-laboratory interactions have used laboratory records as their starting point and been based in hospitals in wealthy countries. The strength of our study is that it was prospective, based in a developing country and used direct observation. The presence of an observer may have altered the behaviour of clinicians but would have resulted in better adherence to the guidelines. Many of the decisions about appropriateness of test requesting and usage were subjective, but we have tried to standardise this by using national guidelines, having a single observer and using a cross-checking process for difficult categorisations.

\section{Challenges for improving laboratory utilisation in poorer countries}

This study demonstrates the extent of, and reasons for, ineffective utilisation of laboratory services in a country with limited resources. Other studies have suggested that some of the markers we used in this study, such as the appropriateness of the test repertoire, could be used as surrogate markers for assessing the effectiveness of the laboratory's contribution to clinical outcomes. ${ }^{15}$ In wealthy countries, convincing clinicians of the need to better integrate laboratory and clinical services, linked to interventions to alter behaviour, was more beneficial than didactic teaching in improving laboratory utilisation. ${ }^{16}{ }^{17}$

Our findings raise several difficult issues for policy makers in developing countries. Although clinical guidelines make specific recommendations about use of laboratory tests, it may not be cost effective to provide all these tests unless training, supervision and auditing systems are established to ensure that they are used effectively by clinicians. Where there is significant underutilisation of laboratory services, any attempt to improve test requesting will have major implications for laboratory budgets, space, staffing and training, as well as on clinical

\section{Take-home messages}

- It may not be cost effective to provide all the tests recommended in clinical guidelines unless training, supervision and auditing systems are established to ensure that tests are used effectively by clinicians.

- Efforts to improve significant underutilisation of laboratory services have implications for budgets, space, staffing and training.

- Policy makers in poorer countries need achieve a balance between the cost of tests and their clinical usefulness; this may mean focusing efforts on providing good quality, high demand tests at the expense of less frequently requested ones.

capacity to obtain samples and use results. Policy makers will need achieve a balance between the cost of tests and their clinical usefulness; ${ }^{11}$ where resources are very limited this may mean focusing efforts on providing good quality, high demand tests at the expense of less frequently requested ones.

Acknowledgements: We would like to acknowledge Charles Gilks, Katherine Floyd, Catherine Mundy and Andrew Kashoti (deceased) for their invaluable assistance with this study.

Funding: This study was funded by the Department for International Development, UK, through the HIV/AIDs Knowledge Programme of the Liverpool School of Tropical Medicine. The Department for International Development had no role in study design, in the collection, analysis and interpretation of data, in the writing of this paper, or in the decision to submit the paper for publication, and it accepts no responsibility for the information or views expressed.

\section{Competing interests: None.}

Ethics approval: Ethics approval was obtained from the Liverpool School of Tropical Medicine, UK, and the Ministry of Health and Population, Malawi.

Provenance and peer review: Not commissioned; not externally peer reviewed.

\section{REFERENCES}

1. Moore RA. Current perspectives in evidence-based laboratory medicine. Br J Biomed Sci 1999;56:226-33.

2. van Walraven C, Naylor CD. Do we know what inappropriate laboratory utilization is? A systematic review of laboratory clinical audits. JAMA 1998;280:550-8.

3. Lewandrowski K. Managing utilization of new diagnostic tests. Clin Leadersh Manag Rev 2003;17:318-24.

4. Wong ET. Cost-effective use of laboratory tests: a joint responsibility of clinicians and laboratorians. Clin Lab Med 1985;5:665-72.

5. Wu AH. Reducing the inappropriate utilisation of clinical laboratory tests. Conn Med 1997;61:15-21.

6. Wennberg DE, Kellett MA, Dickens JD, et al. The association between local diagnostic testing intensity and invasive cardiac procedures. JAMA 1996;275:1161-4.

7. Verrilli D, Welch G. The impact of diagnostic testing on therapeutic interventions. JAMA 1996;275:1189-91.

8. Lundberg GD. The need for an outcomes research agenda for clinical laboratory testing. JAMA 1998;280:565-6.

9. Mundy CJF, Bates I, Nkhoma W, et al. The operation, quality and costs of a district hospital laboratory service in Malawi. Trans R Soc Trop Med Hyg 2003;97:403-8.

10. Ministry of Health, Malawi. Malawi standard treatment guidelines. 2nd edn. Lilongwe: Capital Printing Press, 1993.

11. Bold AM, Corrin B. Use and abuse of clinical chemistry in surgery. BMJ 1965;2:1051-2

12. Mayer M, Wilkinson I, Heikkinen R, et al. Improved laboratory tests selection and enhanced perception of test results as tools for cost-effective medicine. Clin Chem Lab Med 1998;36:683-90.

13. Zikusooka CM, McIntyre D, Barnes Kl. Should countries implementing an artemisinin-based combination malaria treatment policy also introduce rapid diagnostic tests? Malar J 2008; 7:176.

14. Barnish G, Iboro J, Bates I. Newer drug combinations for malaria may be impractical unless diagnostic accuracy can be improved. BMJ 2004;328:1511-2.

15. Waise A, Plebani M. Which surrogate marker can be used to assess the effectiveness of the laboratory and its contribution to clinical outcome? Ann Clin Biochem 2001;38:589-95.

16. Davis D, O'Brien MAT, Freemantle N, et al. Impact of formal continuing medical education: do conferences, workshops, rounds, and other traditional continuing education activities change physician behavior or health care outcomes? JAMA 1999;282:867-74.

17. Winkens R, Geert-Jan D. Rational, cost effective use of investigations in clinical practice. BMJ 2002;324:783-5. 